\title{
Effects of long-range disorder and electronic interactions on the optical properties of graphene quantum dots
}

\author{
A. Altıntaş, K. E. Çakmak, and A. D. Güçlü \\ Department of Physics, Izmir Institute of Technology, IZTECH, TR-35430 Izmir, Turkey \\ (Received 7 September 2016; revised manuscript received 18 January 2017; published 31 January 2017)
}

\begin{abstract}
We theoretically investigate the effects of long-range disorder and electron-electron interactions on the optical properties of hexagonal armchair graphene quantum dots consisting of up to 10806 atoms. The numerical calculations are performed using a combination of tight-binding, mean-field Hubbard, and configuration interaction methods. Imperfections in the graphene quantum dots are modeled as a long-range random potential landscape, giving rise to electron-hole puddles. We show that, when the electron-hole puddles are present, the tight-binding method gives a poor description of the low-energy absorption spectra compared to mean-field and configuration interaction calculation results. As the size of the graphene quantum dot is increased, the universal optical conductivity limit can be observed in the absorption spectrum. When disorder is present, the calculated absorption spectrum approaches the experimental results for isolated monolayers of graphene sheets.
\end{abstract}

DOI: 10.1103/PhysRevB.95.045431

\section{INTRODUCTION}

Graphene, a single layer of carbon atoms arranged in a twodimensional honeycomb lattice [1,2], has attracted enormous research interest due to its superior electronic conductivity [2-5], mechanical strength [6-8], thermal conductivity [8], and unique optical properties $[9,10]$. Moreover, the electronic and optical properties of graphene can be manipulated at the nanoscale in a desired way by controlling the lateral size, shape, type of edge, doping level, and the number of layers in graphene nanostructures [11-16]. Among those various nanostructures of graphene, graphene quantum dots (GQDs) [17-32] offer a possibility to simultaneously control the electronic, magnetic, and optical functionalities in a single material.

GQDs are classified according to their edge character since the edges play an important role in determining the electronic, optical, and magnetic properties of GQDs [19-28]. In particular, armchair and zigzag edges are the most stable edge structures $[16,23,24]$ while GQDs with zigzag edges are found to exhibit unusual magnetic [25-27] and optical [28-32] properties due to the presence of a degenerate band of states at the Fermi level. On the other hand, armchair edges do not lead to a degenerate band of states at the Fermi level, and hence can be used as a small model of bulk graphene which does not have edge states [33].

The properties of graphene nanostructures fabricated and observed upon substrates [34,35] may become affected by imperfections due to the environment and become disordered. In particular, if the disorder has a long-range character, it can lead to charge localizations as electron-hole puddles [36-39]. For instance, the magnetic properties of graphene nanoribbons are found to be strongly dependent on long-range impurities [40]. In addition, the role of electron-hole puddles on the formation of Landau levels in a graphene double quantum dot was investigated experimentally by Chiu et al. [41].

On the other hand, a striking optical property of graphene is the universal optical conductivity (UOC), which can be identified as the explicit manifestation of light and matter interactions [42-44]. The experimental observation of UOC for a graphene sheet seems to indicate that optical properties are robust against imperfections, although significant deviations from UOC at lower energies were observed $[45,46]$. To our knowledge, a detailed theoretical investigation of the combined effects of long-range disorder and electron-electron interactions on the optical properties of graphene quantum dots is still lacking.

In this paper, we investigate theoretically the electronic and optical properties of medium and large sized hexagonal armchair GQDs consisting of up to 10806 atoms to understand the role of long-ranged disorder on the optical properties. Our main contribution involves the inclusion of electron-electron interactions within mean-field and many-body configuration interaction approaches. We show that the electron-electron interactions play a significant role in redistributing electronhole puddles, thus strongly affecting the optical properties. We also investigate the large size limit of the GQDs as compared to the optical properties of bulk graphene [44-46] and show that UOC can be observed in GQDs with a diameter of $18 \mathrm{~nm}$.

The paper is organized as follows. In Sec. II, we describe our model Hamiltonian including an electron-electron interaction and random potential term, and the computational methods that we use in order to compute the optical properties of hexagonal armchair GQDs. The computational results on the electronic and optical properties are presented in Sec. III. Finally, Sec. IV provides a summary and conclusion.

\section{METHOD AND MODEL}

In the tight-binding (TB) approach, the one electron states of GQD can be written as a linear combination of $p_{z}$ orbitals on every carbon atom since the $s, p_{x}$, and $p_{y}$ orbitals are considered to be mainly responsible for the mechanical stability of graphene. Then, within the mean-field extended Hubbard approach, the Hamiltonian can be written as

$$
\begin{aligned}
H_{\mathrm{MFH}}= & \sum_{i j \sigma}\left(t_{i j} c_{i \sigma}^{\dagger} c_{j \sigma}+\text { H.c. }\right)+U \sum_{i \sigma}\left(\left\langle n_{i \sigma}\right\rangle-\frac{1}{2}\right) n_{i \bar{\sigma}} \\
& +\sum_{i j \sigma} V_{i j}\left(\left\langle n_{j}\right\rangle-1\right) n_{i \sigma}+\sum_{i \sigma} V_{\mathrm{imp}}(i) c_{i \sigma}^{\dagger} c_{i \sigma},
\end{aligned}
$$



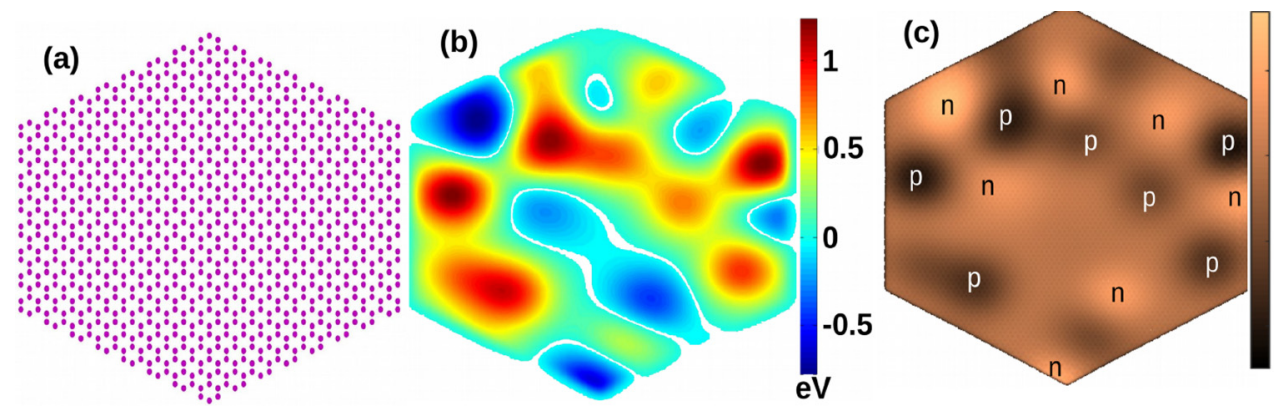

4.86

FIG. 1. (a) Lattice structure of a hexagonal armchair-edged GQD. (b) Impurity potential landscape. (c) Electron-hole puddle formation achieved by the MFH model where local charge accumulation is indicated as $n$ and $p$ puddles.

where the first term represents the TB Hamiltonian and $t_{i j}$ are the hopping parameters given by $t_{\mathrm{nn}}=-2.8 \mathrm{eV}$ for nearest neighbors and $t_{\mathrm{nnn}}=-0.2 \mathrm{eV}$ for next-nearest neighbors [47]. The $c_{i \sigma}^{\dagger}$ and $c_{i \sigma}$ are creation and annihilation operators for an electron at the $i$ th orbital having spin $\sigma$, respectively. The expectation values of the electron densities are represented by $\left\langle n_{i \sigma}\right\rangle$. The second and third terms represent on-site and long-range Coulomb interactions, respectively. We take the on-site interaction parameter as $U=16.522 / \kappa \mathrm{eV}$ and longrange interaction parameters $V_{i j}=8.64 / \kappa$ and $V_{i j}=5.33 / \kappa$ for the first- and second-nearest neighbors with an effective dielectric constant $\kappa=6$, [48], respectively. The distantneighbor interaction is taken to be $1 / d_{i j} \kappa$ and the interaction matrix elements are obtained from numerical calculations by using Slater $\pi_{z}$ orbitals [49]. The last term corresponds to the impurity potential $V_{\text {imp }}(i)$ that accounts for substrate effects.

After diagonalizing the mean-field Hubbard (MFH) matrix self-consistently by starting with TB orbitals, we obtain the Hubbard quasiparticle spectrum which has a fully occupied valence band and a completely empty conduction band. Next, in order to take into account the two-body configuration interactions (CIs), the excitonic correlation effects of the electron-hole, we solve the many-body Hamiltonian for a hole and an electron,

$$
\begin{aligned}
H_{e h}= & \sum_{p^{\prime} \sigma} \epsilon_{p^{\prime}} b_{p^{\prime} \sigma}^{\dagger} b_{p^{\prime} \sigma}-\sum_{p, \sigma} \epsilon_{p} h_{p \sigma}^{\dagger} h_{p \sigma}-\sum_{\substack{p^{\prime}, q, r, s^{\prime} \\
\sigma, \sigma^{\prime}}}\left\{\left\langle r p^{\prime}|V| s^{\prime} q\right\rangle\right. \\
& \left.-\left(1-\delta_{\sigma \sigma^{\prime}}\right)\left\langle r p^{\prime}|V| q s^{\prime}\right\rangle\right\} b_{p^{\prime} \sigma}^{\dagger} h_{q \sigma^{\prime}}^{\dagger} h_{r \sigma^{\prime}} b_{s^{\prime} \sigma} \\
& +\sum_{\substack{p^{\prime}, q, r, s^{\prime} \\
\sigma, \bar{\sigma}}}\left\langle r p^{\prime}|V| q s^{\prime}\right\rangle b_{p^{\prime} \bar{\sigma}}^{\dagger} h_{q \sigma}^{\dagger} h_{r \bar{\sigma}} b_{s^{\prime} \sigma} .
\end{aligned}
$$

Here, the first two terms describe the electron and hole quasiparticle energies obtained from the mean-field calculations, the third term describes the electron-hole Coulomb attraction, and the fourth and fifth terms represent the electron-hole exchange interactions. Indices with a prime denote electron states and without a prime denote hole states. The two-body electronhole scattering matrix elements are calculated from two-body on-site and long-range Coulomb matrix elements [18].

In this paper, we consider three different sizes of hexagonal armchair GQDs [see, for example, Fig. 1(a)] consisting of 1014,5514 , and 10806 atoms and having widths of 5, 13, and $18 \mathrm{~nm}$, respectively.
In order to model the long-range disorder due to charge impurities caused by substrate effects, we use a superposition of Gaussian electrostatic potentials $V_{\text {imp }}$ which are determined randomly to have a smooth potential landscape [see Fig. 1(b)] on the GQD. The impurity potential is written as

$$
V_{\text {imp }}\left(r_{i}\right)=\sum_{k} V_{k} \exp \left[-\frac{\left(\vec{r}_{i}-\vec{R}_{k}\right)^{2}}{2 \sigma^{2}}\right],
$$

where $V_{k}$ is chosen to be the potential peak value which is randomly generated between $-V_{\max }<V_{k}<V_{\max }$ values for an impurity at $R_{k}$, characterizing the strength of the disorder. For most of the calculations, we take $V_{\max }=t_{\mathrm{nn}} / 3$, giving a medium disorder strength. However, the effect of strong $\left(V_{\max }=t_{\mathrm{nn}}\right)$ and weak $\left(V_{\max }=t_{\mathrm{nn}} / 5\right)$ disorder is also investigated (see Fig. 5). The width of the potential $\sigma$ is determined to be ten times the lattice constant in order to simulate long-range lattice scatterers [36]. For $5 \mathrm{~nm}$ (1014 atoms), $13 \mathrm{~nm}$ (5514 atoms), and $18 \mathrm{~nm}$ (10 806 atoms) wide GQDs, respectively 4, 20, and 40 source points of impurities are randomly created to have approximately similar source point densities (but different forms of distribution of the source
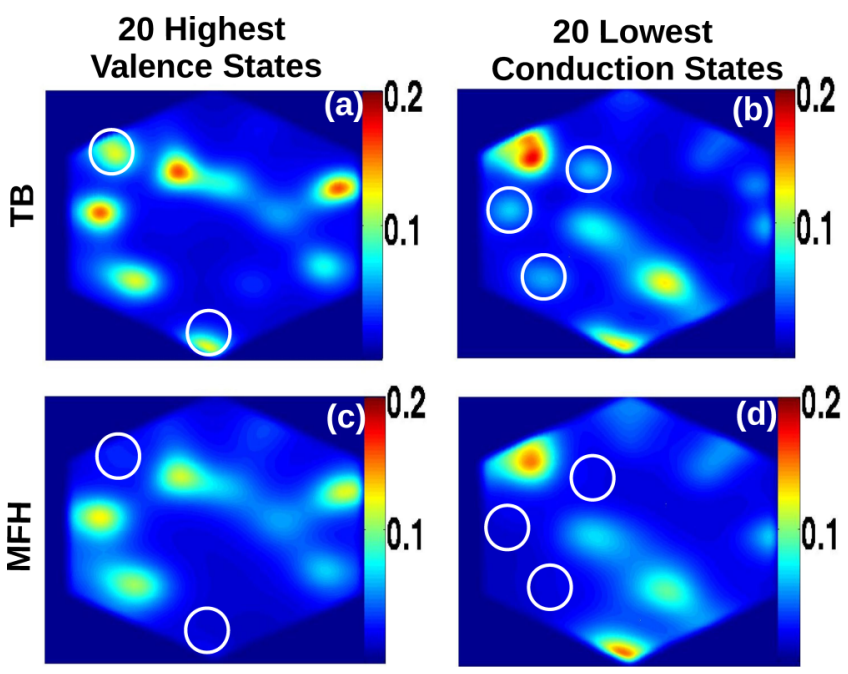

FIG. 2. Electronic density corresponding to the 20 highest valence states (left panels), and the 20 lowest conduction states (right panels), obtained from TB (upper panels) and MFH (lower panels) models of the structure of $18 \mathrm{~nm}$ width size. Electron-electron interactions wash out abnormal localized states near the Fermi level, as indicated by white circles. 
points) for each GQD. Moreover, we considered five different randomly chosen potential configurations for each QD size. The main effect of long-range disorder on the electronic densities is the formation of electron-hole puddles [36,40], as seen in Fig. 1(c), obtained by subtraction of the positive background charge from the MFH electronic density. The effect of the electron-hole puddles on the optical properties will be investigated below using the TB, MFH, and CI approaches.

The interactions of GQD's electrons with photons are evaluated within the electric dipole approximation by the interaction Hamiltonian $H_{\text {int }}=\mathbf{E} \cdot \mathbf{r}$, where $\mathbf{E}$ is the photon's electric field and $\mathbf{r}$ is the electron's position. Hence, one can obtain an absorption spectrum by using the light-matter interaction, which is described as

$$
A(\omega)=\sum_{f} \frac{4 \pi^{2} \alpha E_{f i}|\langle f|\mathbf{r}| i\rangle|^{2} \delta\left(\hbar \omega-E_{f i}\right)}{\text { Area }}
$$

where $\alpha$ is the fine structure constant, Area is the area of the QD, $E_{f i}$ is the difference between the initial and final energies, $\langle f|\mathbf{r}| i\rangle$ denotes the dipole matrix element, and $|i\rangle$ and $|f\rangle$ denote the initial and final occupied molecular orbitals, respectively, obtained by the TB and MFH models.
On the other hand, we obtain an absorption spectrum which includes many-body correlations as

$$
A(\omega)=\sum_{f} \frac{4 \pi^{2} \alpha E_{f i}\left|\left\langle f\left|\mathbf{P}^{\dagger}\right| \mathrm{gs}\right\rangle\right|^{2} \delta\left(\hbar \omega-E_{f i}\right)}{\text { Area }},
$$

where $\alpha$ is the fine structure constant, Area is the area of the $\mathrm{QD}, E_{f i}$ is the difference between the initial (ground state) and final energies of the exciton, and $\mathbf{P}^{\dagger}$ annihilates a photon and adds an exciton to the ground state of the GQD. The final excitonic state $|f\rangle$ is obtained from CI calculations, and $|g s\rangle$ is the ground state.

\section{RESULTS}

In Fig. 2, we investigate the electronic densities corresponding to the 20 lowest conduction and 20 highest valence states obtained from TB and MFH calculations for the largest GQD structure that we studied, which has 10806 atoms, giving a width of $18 \mathrm{~nm}$ [see the corresponding potential landscape in Fig. 1(b)]. We note that we repeated all the calculations for five different random potential landscapes (for each QD size) and observed similar behaviors. In the TB results, in addition to valence states accumulated around the peaks and conduction states around the troughs [see Figs. 2(a) and 2(b)] as expected, we also observe abnormal valence states around the troughs
1014 atoms
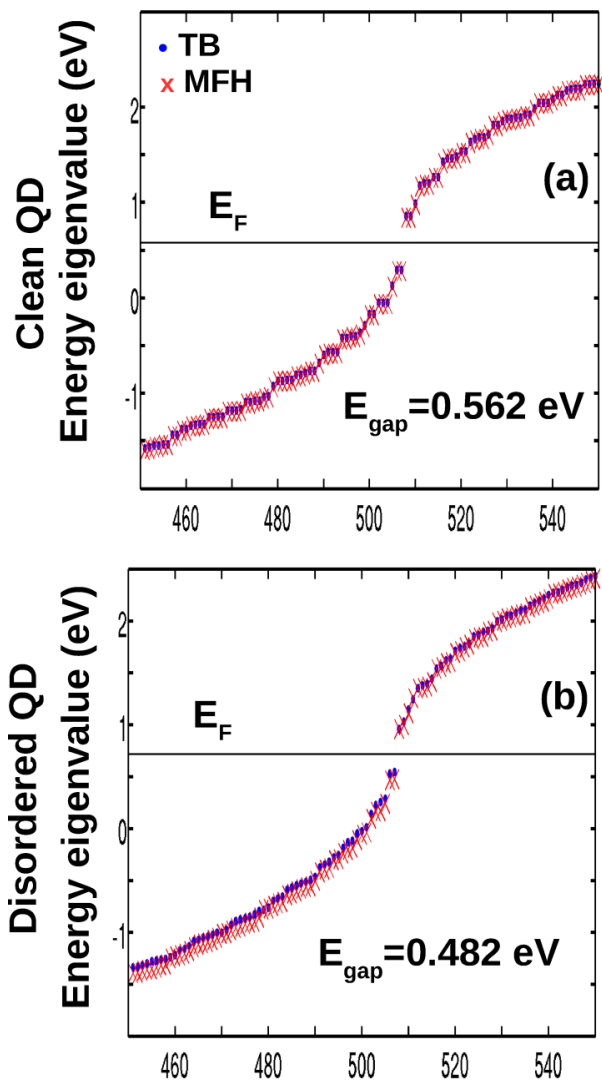

5514 atoms
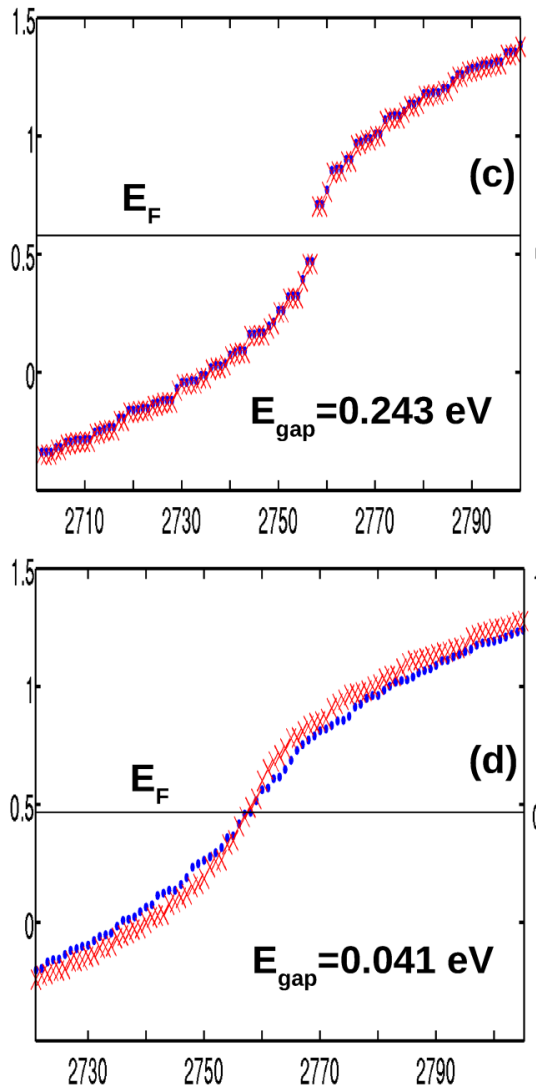

Eigenvalue index

FIG. 3. Energy spectra for clean (upper panels) and disordered (lower panels) GQDs obtained by TB and MFH. The Fermi energy level $E_{F}$ is determined to be in the midpoint between the valence and conduction band. 
and conduction states around the peaks [shown as circles, to be compared with Fig. 1(b)]. In fact, those abnormal states are an artifact of the TB method which is better suited for systems with homogeneous and neutral charge distributions. In our system, the charge density fluctuates strongly due to random disorder and the energy gap between the valence and conduction states is not large enough to protect the hole states from mixing with the electron states. Thus, a meanfield correction to the TB method must be included. Indeed, when electron-electron interactions are included through $\mathrm{MFH}$ calculations, electronic density fluctuations are reduced in almost all areas of the QD and the abnormal localized states are washed out [see Figs. 2(c) and 2(d)]. A similar behavior was also observed in graphene nanoribbons [40]. As we will see, the rearrangement of electron-hole puddles through electronic interactions has an important effect on the optical properties.

The energy spectra of clean (upper panels) and disordered (lower panels) GQDs having a width size of $5 \mathrm{~nm}$ (1014 atoms), $13 \mathrm{~nm}$ (5514 atoms), and $18 \mathrm{~nm}$ (10 806 atoms) obtained by the TB and MFH models are shown in Fig. 3. For each case, the energy gap $E_{\text {gap }}$ between the lowest unoccupied conduction state and the highest occupied valence state obtained from the MFH calculations is indicated as well. As expected, $E_{\text {gap }}$ decreases more rapidly as a function of size when impurities are present. More interestingly, however, for larger sized disordered GQDs, the difference between the TB and MFH spectra becomes pronounced, indicating that when charge inhomogeneities (due to electron-puddle formation) are present, it is important to include the effects of electronic interactions. A similar behavior was also observed for other random potential configurations that we have tested.

Figure 4 shows absorption spectra curves corresponding to the GQDs considered in Fig. 3 for energies up to $3 \mathrm{eV}$. The absorption spectra are calculated using Eqs. (4) and (5) with a Gaussian broadening $(0.1 \mathrm{eV})$ of the delta functions in order to obtain continuous curves, within TB (red curve), $\mathrm{MFH}$ (green curve), and CI (blue curve) approaches. The UOC is indicated by a black line as a reference. For clean GQDs, there is no noticeable difference between the TB and MFH results, consistent with the results in Fig. 3. We note that, as the system size increases, absorption curves approach the UOC value at low energies, until a sudden drop occurs due to finite size effects. For the CI calculations, the 100 highest valence and 100 lowest conduction states were included to form a many-body basis set of 10000 excitonic states, to ensure convergence for energies up to $0.75 \mathrm{eV}$. As seen from Figs. 4(a) and 4(b), the main effect of the excitonic correlations is to redshift the absorption spectrum [32], followed by a slight decrease in the peak value. For GQDs larger than 13 nm (5514 atoms), it was not possible to calculate the $\mathrm{CI}$ absorption spectrum due to computational limits.
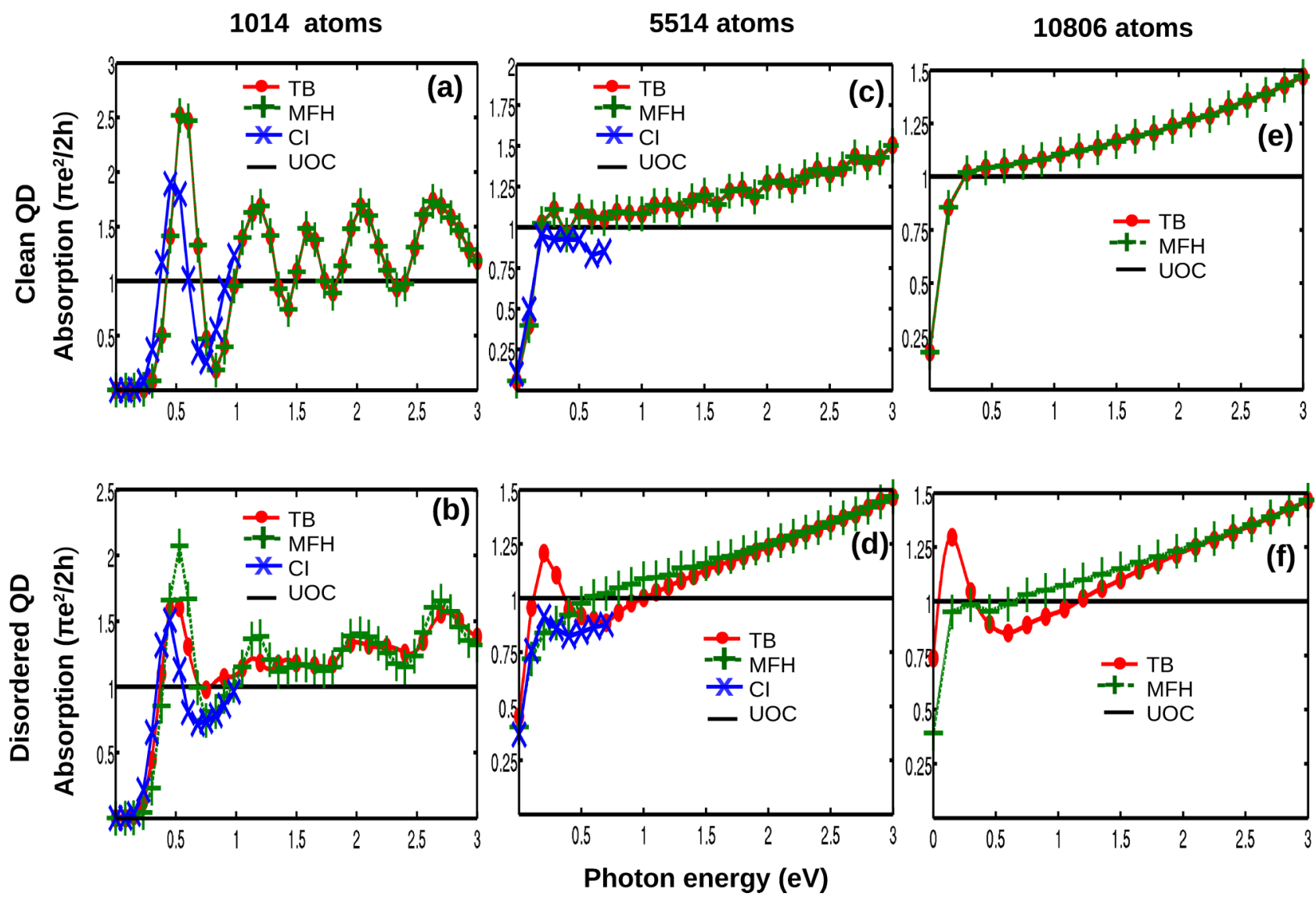

FIG. 4. Absorption spectrum for clean (upper panels) and disordered (lower panels) GQDs obtained by TB, MFH, and excitonic effects with the CI model. UOC is indicated by a black line. In clean GQDs, as the sizes of the GQDs increase, a plateau develops near the UOC at low energies, before a sudden drop occurs due to finite size effects. 


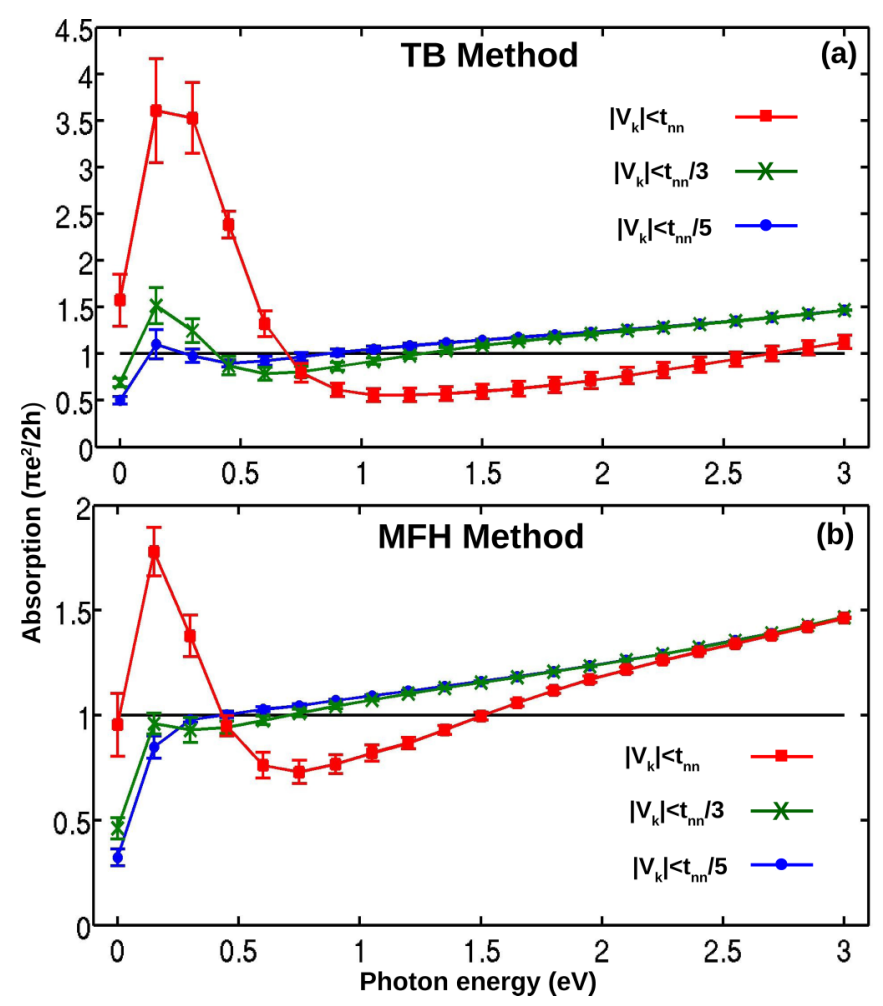

FIG. 5. Average absorption spectrum curves (each curve corresponds to an average of five different configurations) for three different impurity potential peaks obtained by TB (upper panel) and MFH (lower panel) methods with estimated error bars for the structure containing 10806 atoms. UOC is indicated by a black line.

When disorder is present, we observe a dramatic difference between the TB and MFH results, shown in Figs. 4(b), 4(d), and 4(f). This is mainly due to the redistribution of electronhole puddles discussed in Fig. 2. For the medium and large sized GQDs without electronic interactions, in TB calculations, both electrons and hole puddles may be present at the same locations, giving rise to a stronger electric dipole coupling, and thus higher absorption values on average at lower energies. Note that the situation is different for the GQD with 1014 atoms, since the puddle formation is much less well defined as the size of the QD is reduced, and the specific form of the disorder landscape has a bigger role. For medium sized GQDs, however, a disorder peak reappears at low energies when excitonic correlations are taken into account. This is due to the fact that excitonic interactions rearrange the electron and hole distributions within the disorder troughs and peaks, as we discuss below in Fig. 6. We note that the CI results obtained for the disordered GQD with 5514 atoms are consistent with the experimental results for graphene sheets $[9,45,46]$.

To see the effects of various impurity potential strengths on the absorption spectrum obtained by TB and MFH methods [see Figs. 5(a) and 5(b)], we compare spectrum curves (each spectrum curve corresponds to an average of five different samples shown with error bars having widths of twice the standard error) containing three different impurity potential strength peak values of $t_{\mathrm{nn}}$ (red curve), $t_{\mathrm{nn}} / 3$ (green curve), and $t_{\mathrm{nn}} / 5$ (blue curve), for the largest QD structure. For the strong impurity potential strength $\left(\left|V_{k}\right|<t_{\mathrm{nn}}\right)$, both $\mathrm{TB}$ and $\mathrm{MFH}$
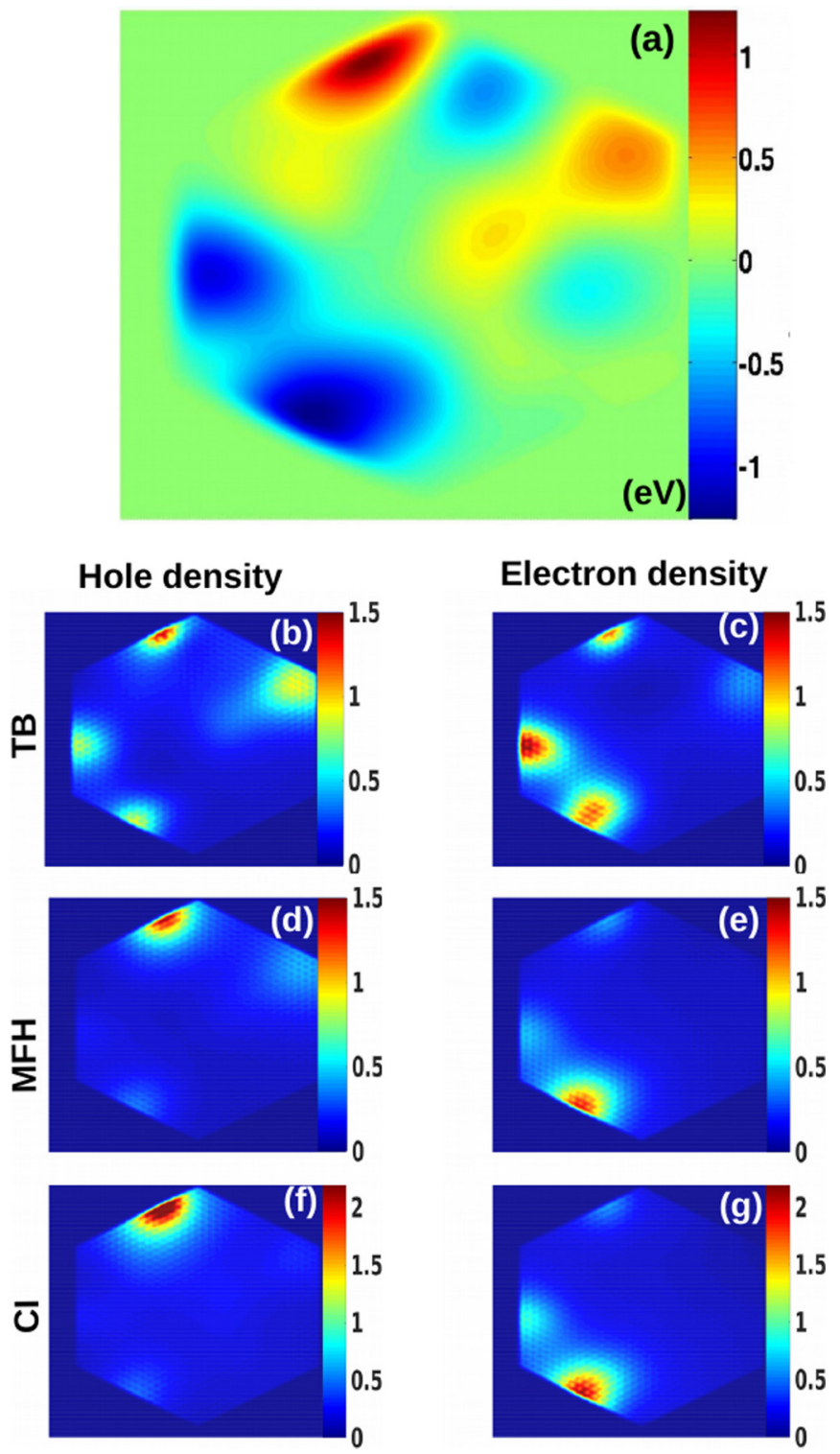

FIG. 6. (a) Impurity potential for the structure containing 5514 atoms. (b)-(g) show the corresponding electron and hole densities weighted with absorption probabilities in the energy range between 0 and $0.3 \mathrm{eV}$ obtained by the TB, MFH, and CI models, respectively.

results deviate significantly from the UOC line, indicating that the system is in a strongly nonperturbative regime, and mean-field electron interactions are not sufficiently strong to wash out the impurity peak. However, for a medium potential strength $\left(\left|V_{k}\right|<t_{\mathrm{nn}} / 3\right)$ and a small potential strength $\left(\left|V_{k}\right|<\right.$ $\left.t_{\mathrm{nn}} / 5\right)$, the low-energy absorption obtained from MFH remains always below the UOC line within our error bars.

In order to investigate further the effect of excitonic correlations, in Fig. 6 we plot the electron and hole densities weighted with absorption probabilities in the energy range between 0 and $0.3 \mathrm{eV}$ for the 5514 atom GQD, obtained from the TB, MFH, and CI calculations. As discussed earlier, mean-field interactions smooth the puddles so that excitonic hole states are now localized only on the peaks, and the electron states are localized on the troughs, as seen in Figs. 6(d) and $6(\mathrm{e})$. On the other hand, the correlations have a less 
dramatic effect on the density distribution, but the electron states are now slightly more localized on a potential trough that is closer to the hole puddle [see Figs. 6(f) and 6(g)]. Indeed, the electron-hole attraction is favored in the CI calculations, minimizing the average distance between the electron and the hole, thus increasing the electric dipole strength and the absorption at lower energies.

\section{CONCLUSIONS}

In conclusion, we have investigated the electronic and optic properties of three different sizes of clean and disordered hexagonal armchair-edged GQDs by applying tight-binding, mean-field Hubbard, and configuration interaction models. Long-ranged disorder give rise to the formation of electronhole puddles, which are, however, poorly described by the tight-binding model alone. Electronic interactions in the meanfield picture reorganize the electron-hole puddles, strongly affecting the dipole moments between the low-energy states in the electronic spectrum. Hence, the inclusion of electronic interactions is found to be important in order to correctly describe the optical properties. As the system size is increased to $18 \mathrm{~nm}$, the absorption spectra obtained from the configuration interaction method approach the experimental results, leading to the observation of universal optical conductivity $[45,46]$.
[1] P. R. Wallace, Phys. Rev. 71, 622 (1947).

[2] K. S. Novoselov, A. K. Geim, S. V. Morozov, D. Jiang, Y. Zhang, S. V. Dubonos, I. V. Grigorieva, and A. A. Firsov, Science 306, 666 (2004).

[3] K. S. Novoselov, A. K. Geim, S. V. Morozov, D. Jiang, M. I. Katsnelson, I. V. Grigorieva, S. V. Dubonos, and A. A. Firsov, Nature (London) 438, 197 (2005).

[4] Y.Zhang, Y.-W. Tan, H. L. Stormer, and P. Kim, Nature (London) 438, 201 (2005).

[5] A. Rycerz, J. Tworzydło, and C. W. J. Beenakker, Nat. Phys. 3, 172 (2007).

[6] C. Lee, X. Wei, J. W. Kysar, and J. Hone, Science 321, 385 (2008).

[7] D. A. Dikin, S. Stankovich, E. J. Zimney, R. D. Piner, G. H. B. Dommett, G. Evmenenko, S. T. Nguyen, and R. S. Ruoff, Nature (London) 448, 457 (2007).

[8] G. Xin, T. Yao, H. Sun, S. M. Scott, D. Shao, G. Wang, and J. Lian, Science 349, 1083 (2015).

[9] K. F. Mak, M. Y. Sfeir, Y. Wu, C. H. Lui, J. A. Misewich, and T. F. Heinz, Phys. Rev. Lett. 101, 196405 (2008).

[10] Y. Zhang, T. T. Tang, C. Girit, Z. Hao, M. C. Martin, A. Zettl, M. F. Crommie, Y. R. Shen, and F. Wang, Nature (London) 459, 820 (2009).

[11] X. Li, X. Wang, L. Zhang, S. Lee, and H. Dai, Science 319, 1229 (2008).

[12] J. Cai, P. Ruffieux, R. Jaafar, M. Bieri, T. Braun, S. Blankenburg, M. Muoth, A. P. Seitsonen, M. Saleh, X. Feng, K. Müllen, and R. Fasel, Nature (London) 466, 470 (2010).

[13] M. Treier, C. A. Pignedoli, T. Laino, R. Rieger, K. Müllen, D. Passerone, and R. Fasel, Nat. Chem. 3, 61 (2011).

[14] M. L. Mueller, X. Yan, J. A. McGuire, and L. S. Li, Nano Lett. 10, 2679 (2010).

[15] Y. Morita, S. Suzuki, K. Sato, and T. Takui, Nat. Chem. 3, 197 (2011).

[16] T. Wassmann, A. P. Seitsonen, A. M. Saitta, M. Lazzeri, and F. Mauri, Phys. Rev. Lett. 101, 096402 (2008).

[17] J. Shen, Y. Zhu, X. Yang, and C. Li, Chem. Commun. 48, 3686 (2012).

[18] A. D. Güçlü, P. Potasz, M. Korkusinski, and P. Hawrylak, Graphene Quantum Dots (Springer, Berlin, 2014).

[19] K. A. Ritter and J. W. Lyding, Nat. Mater. 8, 235 (2009).

[20] J. Peng, W. Gao, B. K. Gupta, Z. Liu, R. R. Aburto, L. Ge, L. Song, L. B. Alemany, X. Zhan, G. Gao, S. A. Vithayathil,
B. A. Kaipparettu, A. A. Marti, T. Hayashi, J. J. Zhu, and P. M. Ajayan, Nano Lett. 12, 844 (2012).

[21] B. Trauzettel, D. V. Bulaev, D. Loss, and G. Burkard, Nat. Phys. 3, 192 (2007).

[22] B. D. Gerardot, D. Brunner, P. A. Dalgarno, P. Öhberg, S. Seidl, M. Kroner, K. Karrai, N. G. Stoltz, P. M. Petroff, and R. J. Warburton, Nature (London) 451, 441 (2008).

[23] M. Zarenia, A. Chaves, G. A. Farias, and F. M. Peeters, Phys. Rev. B 84, 245403 (2011).

[24] O. Voznyy, A. D. Güçlü, P. Potasz, and P. Hawrylak, Phys. Rev. B 83, 165417 (2011).

[25] P. Potasz, A. D. Güçlü, A. Wójs, and P. Hawrylak, Phys. Rev. B 85, 075431 (2012).

[26] A. D. Güçlü, P. Potasz, and P. Hawrylak, Phys. Rev. B 84, 035425 (2011).

[27] A. D. Güçlü, P. Potasz, O. Voznyy, M. Korkusinski, and P. Hawrylak, Phys. Rev. Lett. 103, 246805 (2009).

[28] A. D. Güçlü, P. Potasz, and P. Hawrylak, Phys. Rev. B 82, 155445 (2010).

[29] T. Basak, H. Chakraborty, and A. Shukla, Phys. Rev. B 92, 205404 (2015).

[30] C. Sun, F. Figge, I. Ozfidan, M. Korkusinski, X. Yan, L. Li, P. Hawrylak, and J. A. McGuire, Nano Lett. 15, 5472 (2015).

[31] Y. Li, H. Shu, S. Wang, and J. Wang, J. Phys. Chem. C 119, 4983 (2015).

[32] I. Ozfidan, A. D. Güçlü, M. Korkusinski, and P. Hawrylak, Phys. Status Solidi RRL 10, 102 (2016).

[33] T. Yamamoto, T. Noguchi, and K. Watanabe, Phys. Rev. B 74, 121409 (2006).

[34] L. Liao, Y. C. Lin, M. Bao, R. Cheng, J. Bai, Y. Liu, Y. Qu, K. L. Wang, Y. Huang, and X. Duan, Nature (London) 467, 305 (2010).

[35] Y. M. Lin, C. Dimitrakopoulos, K. A. Jenkins, D. B. Farmer, H. Y. Chiu, A. Grill, and Ph. Avouris, Science 327, 662 (2010).

[36] Y. Zhang, V. W. Brar, C. Girit, A. Zettl, and M. F. Crommie, Nat. Phys. 5, 722 (2009).

[37] J. Martin, N. Akerman, G. Ulbricht, T. Lohmann, J. H. Smet, K. V. Klitzing, and A. Yacoby, Nat. Phys. 4, 144 (2008).

[38] M. Gibertini, A. Tomadin, F. Guinea, M. I. Katsnelson, and M. Polini, Phys. Rev. B 85, 201405 (2012). 
[39] Y. W. Tan, Y. Zhang, K. Bolotin, Y. Zhao, S. Adam, E. H. Hwang, S. Das Sarma, H. L. Stormer, and P. Kim, Phys. Rev. Lett. 99, 246803 (2007).

[40] H. U. Özdemir, A. Altıntaş, and A. D. Güçlü, Phys. Rev. B 93, 014415 (2016).

[41] K. L. Chiu, M. R. Connolly, A. Cresti, J. P. Griffiths, G. A. C. Jones, and C. G. Smith, Phys. Rev. B 92, 155408 (2015).

[42] R. R. Nair, P. Blake, A. N. Grigorenko, K. S. Novoselov, T. J. Booth, T. Stauber, N. M. R. Peres, and A. K. Geim, Science 320, 1308 (2008).

[43] A. B. Kuzmenko, E. van Heumen, F. Carbone, and D. van der Marel, Phys. Rev. Lett. 100, 117401 (2008).
[44] S. Yuan, R. Roldan, H. De Raedt, and M. I. Katsnelson, Phys. Rev. B 84, 195418 (2011).

[45] K. F. Mak, J. Shan, and T. F. Heinz, Phys. Rev. Lett. 106, 046401 (2011).

[46] C. Lee, J. Y. Kim, S. Bae, K. S. Kim, B. H. Hong, and E. J. Choi, Appl. Phys. Lett. 98, 071905 (2011).

[47] S. Reich, J. Maultzsch, C. Thomsen, and P. Ordejon, Phys. Rev. B 66, 035412 (2002).

[48] T. Ando, J. Phys. Soc. Jpn. 75, 074716 (2006).

[49] P. Potasz, A. D. Güçlü, and P. Hawrylak, Phys. Rev. B 82, 075425 (2010). 